\title{
Iterative Method to Solve a Data Completion Problem for Biharmonic Equation for Rectangular Domain
}

\author{
Chakir Tajani, Houda Kajtih, and Ali Daanoun
}

\begin{abstract}
In this work, we are interested in a class of problems of great importance in many areas of industry and engineering. It is the invese problem for the biharmonic equation. It consists to complete the missing data on the inaccessible part from the measured data on the accessible part of the boundary. To solve this ill-posed problem, we opted for the alternative iterative method developed by Kozlov, Mazya and Fomin which is a convergent method for the elliptical Cauchy problems in general. The numerical implementation of the iterative algorithm is based on the application of the boundary element method (BEM) for a sequence of mixed well-posed direct problems. Numerical results are performed for a square domain showing the effectiveness of the algorithm by BEM to produce accurate and stable numerical results
\end{abstract}

AMS Subject Classification (2000). 65N21; 35J30; 65N38 Keywords. inverse problem, biharmonic equation, iterative method, boundary element method

\section{Introduction}

In this paper, we consider an inverse problem for biharmonic equation called data completion problem, which aims at recovering missing conditions on 
some inaccessible part of the boundary (which cannot be evaluated due to the physical difficulties or inaccessibility geometric) from the over-specified boundary data on the remaining part of the boundary.

The biharmonic equation in two dimensions arises naturally in many physical applications. It is a well-known example of a mathematical model governing the interior two-dimensional flow of viscous fluids at small Reynolds numbers, i.e., the Stokes flow or the Kirchhoff theory of plates in elasticity.

The knowledge of the appropriate boundary conditions across the boundary of the domain of the solution results in direct problems of the biharmonic equation which have been widely studied in the literature [1], [2], [3]. Unfortunately, many technical problems are not part of this category. Indeed, the boundary conditions are often incomplete or in the form of the boundary conditions, under-specified and over-specified on different parts of the boundary, or the solution is prescribed at some internal points in the domain. It is the inverse problems known to be generally ill-posed, namely, the existence, uniqueness and stability of their solutions are not always guaranteed [4].

Concerning the inverse problem for the Laplace equation, it has been subject of several works in which many works are focused to develop regularizing methods to overcome the ill-posed aspect of this problem. Among the deterministic method, we find the most ancient of them is the one based on optimization tools introduced by Kohn and Vogelius [5], we mention the method of Quasi-reversibility introduced by Latès since 1960 [6], and recently used by [7],[8], Thikhonv method [9] and the iterative method [10].

Obtaining a numerical solution for the Cauchy problem for the biharmonic equation, which is an extention of the Laplace equation, is very important since the inverse problem is still ill-posed as the solution is not stable with respect to small perturbations in the input data. Hence, the Cauchy problem fo the biharmonic equation has been investigated by [16], [17] and [18]. The aim of this study is to introduce a stable numerical method for obtaining this solution approximately in the case of square domain which is an example of nonsmooth geometry for the biharmonic inverse problem with four conditions on the accessible part of the boundary $\Gamma_{0}$ that are $u, \partial_{n} u, \Delta u$ and $\partial_{n} \Delta u$.

Having distinguished the different categories of methods for solving Cauchy problems, we opted for the group of iterative methods that particularly have two advantages over other methods, namely; the simplicity of numerical implementation and the ability to easily consider any physical constraint in the iterative scheme. Among the iterative methods, we find the method of Kozlov and al. [10], that was widely used and studied [11], [12], [13], [14].

To solve the Cauchy problem, the two equations resulting from the factorization of the biharmonic inverse problem are discretised using the boundary element method (BEM). The advantages of this method for linear partial 
differential equations over domain discretisation methods, such as finite difference or finite element method, are well-known [15]. This paper is organized as follows. In section 2, we present the mathematical formulation of the inverse problem for the biharmonic equation with the adapted boundary conditions and its corresponding factorized problem. The iterative algorithm is presented in section 3. The discretization of the well-posed mixed problems by the boundary element method is described in section 4 . Section 5 is devoted to computational aspects and numerical results for typical example for a square domain.

\section{Mathematical formulation}

\subsection{Biharmonic inverse problem}

We consider an open bounded domain $\Omega \subset \mathbb{R}^{2}$ of boundary $\Gamma=\Gamma_{0} \cup \Gamma_{1}$, where $\Gamma_{0}, \quad \Gamma_{1} \neq \emptyset$ and $\Gamma_{0} \cap \Gamma_{1}=\emptyset$.

We consider that the domain $\Omega$ is governed by the biharmonic equation difined by:

$$
\triangle^{2} u=0 \quad \text { in } \Omega
$$

that is equivalent to system of equations:

$$
\left\{\begin{array}{l}
\triangle u=\omega \text { in } \Omega \\
\triangle \omega=0 \text { in } \Omega
\end{array}\right.
$$

This system is a well known example of a mathematical model which model the Stokes flow, in which case the functions $u$ and $\omega$ are called the current function and the swirl of the fluid flow. The biharmonic operator also governs the flexing of thin plates in small displacements.

Concerning the boundary conditions if for example $u$ and its normal derivative $\frac{\partial u}{\partial n}$ or $u$ and $\omega$ or $u$ and $\frac{\partial \omega}{\partial n}$ are prescribed at all points of the boundary $\Gamma=\partial \Omega$, in this case, it is a well-posed direct problem, then the solutions $u$ and $\omega$ can be uniquely identified throughout the domain $\Omega$. However, in many experimental situations, it is not always possible to specify the boundary conditions at all points of the boundary $\Gamma$; but other inside information or limit can be given elsewhere, then the problem is called an inverse problem for the biharmonic equation which is ill-posed. Such as, if on a part of the boundary $\Gamma_{0} \subset \Gamma$ the conditions $u, \frac{\partial u}{\partial n}, \triangle u$, and $\frac{\partial \Delta u}{\partial n}$ are prescribed and the remaining part $\Gamma_{1}=\Gamma \backslash \Gamma_{0}$ has no limit conditions. 
In this paper, we are interested in the mathematical formulation of the inverse problem associated with the biharmonic equation with four boundary conditions defined by $u, \frac{\partial u}{\partial n}, \triangle u$ and $\frac{\partial \Delta u}{\partial n}$ on the accessible part of the boundary $\Gamma_{0}$ and no conditions are specified on the remaining part $\Gamma_{1}$.

Thus, the inverse problem assicieted to a biharmonic equation can be formulated as follows:

$$
\left\{\begin{array}{ccc}
\triangle^{2} u=0 & \text { in } & \Omega \\
u=u_{0} & \text { on } \Gamma_{0} \\
\frac{\partial u}{\partial n}=u_{0}^{\prime} & \text { on } \Gamma_{0} \\
\triangle u=\omega_{0} & \text { on } \Gamma_{0} \\
\frac{\partial \triangle u}{\partial n}=\omega_{0}^{\prime} & \text { on } \Gamma_{0}
\end{array}\right.
$$

The problem consists in solving the Cauchy problem (2.3) that is from the data $u_{0}, u_{0}^{\prime}, \omega_{0}$ and $\omega_{0}^{\prime}$ on the accessible part $\Gamma_{0}$ of the boundary $\Gamma$ recreate the information on all the boundary and thus find the solution $u$ in the interior of the domain.

The problem (2.3) admits a unique solution for a compatible data on the part of the boundary $\Gamma_{0}$.

Thus; we consider that the solution $u$ is in the space $\mathcal{H}_{0}^{2}\left(\triangle^{2}, \Omega\right)$, defined by $[19]$ :

$$
\mathcal{H}_{0}^{2}\left(\triangle^{2}, \Omega\right)=\left\{u \in H^{2}(\Omega) / \triangle^{2} u=0\right\}
$$

Then, the space of compatible data on $\Gamma_{0}$ is defined by:

$$
\begin{aligned}
K\left(\Gamma_{0}\right) & =\left\{\left(u_{0}, u_{0}^{\prime}, \omega_{0}, \omega_{0}^{\prime}\right) \in X\left(\Gamma_{0}\right) / \exists u \in \mathcal{H}_{0}^{2}\left(\triangle^{2}, \Omega\right) \text { and }\left(u, \frac{\partial u}{\partial n}, \triangle u, \frac{\partial \triangle u}{\partial n}\right)_{/ \Gamma_{0}}\right. \\
& \left.=\left(u_{0}, u_{0}^{\prime}, \omega_{0}, \omega_{0}^{\prime}\right)\right\}
\end{aligned}
$$

where;

$$
X\left(\Gamma_{0}\right)=H^{3 / 2}\left(\Gamma_{0}\right) \times H^{1 / 2}\left(\Gamma_{0}\right) \times H^{-1 / 2}\left(\Gamma_{0}\right) \times H^{-3 / 2}\left(\Gamma_{0}\right) .
$$

Remark. It should be noted that other formulations exist with conditions on the accessible part of the boundary [17].

\subsection{The factorised problem}

To solve the problem (2.3), we propose the factorization of the problem in two inverse Cauchy problems associated with the Laplace and Poisson equations. 
In the problem (2.3), we made the change of unknown function:

$$
\triangle u=\omega
$$

Then, the problem becomes:

$$
\left\{\begin{array}{ccc}
\triangle u=\omega & \text { in } & \Omega \\
\triangle \omega=0 & \text { in } & \Omega \\
u=u_{0} & \text { on } & \Gamma_{0} \\
\frac{\partial u}{\partial n}=u_{0}^{\prime} & \text { on } & \Gamma_{0} \\
\omega=\omega_{0} & \text { on } & \Gamma_{0} \\
\frac{\partial \omega}{\partial n}=\omega_{0}^{\prime} & \text { on } & \Gamma_{0}
\end{array}\right.
$$

This problem is composed of two inverse Cauchy problems:

$$
\left\{\begin{array} { l l } 
{ \triangle \omega = 0 } & { \text { in } \Omega } \\
{ \omega = \omega _ { 0 } } & { \text { on } \Gamma _ { 0 } } \\
{ \frac { \partial \omega } { \partial n } = \omega _ { 0 } ^ { \prime } } & { \text { on } \Gamma _ { 0 } }
\end{array} ( P _ { 1 } ) \text { and } \left\{\begin{array}{clll}
\triangle u=\omega & \text { in } \Omega \\
u=u_{0} & \text { on } \Gamma_{0} & \left(P_{2}\right) \\
\frac{\partial u}{\partial n}=u_{0}^{\prime} & \text { on } \Gamma_{0}
\end{array}\right.\right.
$$

with $\left(P_{1}\right)$ is an inverse problem for the Laplace equation and $\left(P_{2}\right)$ is the inverse problem associated with the Poisson equation which can be solved by the iterative algorithm descibed in the next section.

\section{Description of the iterative algorithm}

The resolution of the problem (2.3) is equivalent to the resolution of the two problems $\left(P_{1}\right)$ and $\left(P_{2}\right)$ of $(2.8)$. This will allow us to iteratively solve, by the boundary element method, both direct mixed problems associated with problems $\left(P_{1}\right)$ and $\left(P_{2}\right)$.

The KMF iterative algorithm investigated proposed by [10] is a convergent algorithm for solving elliptic Cauchy problems and is widely used in the case of Cauchy problems with Laplace's and Poisson's equation [11], [13] and [14] and propsed in this work in the case of the biharmonic problem with the specified conditions in (2.3). This algorithm is based on reducing this illposed problem to a sequence of mixed well-posed boundary value problems and consists of the following steps: 
Step 1: Specify an initial guess $\omega_{1}$ and $u_{1}$ on $\Gamma_{1}$.

Step 2: Solve the following mixed well-posed boundary value problems:

$$
\begin{aligned}
& \left\{\begin{array} { l l } 
{ \triangle \omega ^ { ( 0 ) } = 0 } & { \text { in } \Omega } \\
{ \omega ^ { ( 0 ) } = \omega _ { 1 } } & { \text { on } \Gamma _ { 1 } } \\
{ \frac { \partial \omega } { \partial n } ^ { ( 0 ) } = \omega _ { 0 } ^ { \prime } } & { \text { on } \Gamma _ { 0 } }
\end{array} \quad \text { and } \quad \left\{\begin{array}{cl}
\triangle u^{(0)}=\omega^{(0)} & \text { in } \Omega \\
u^{(0)}=u_{1} & \text { on } \Gamma_{1} \\
\frac{\partial u}{\partial n}^{(0)}=u_{0}^{\prime} & \text { on } \Gamma_{0}
\end{array}\right.\right. \\
& \text { to obtain } \frac{\partial \omega^{(0)}}{\partial n}{ }_{\mid \Gamma_{1}}=v_{1} \quad \text { to obtain }\left.\frac{\partial u^{(0)}}{\partial n}\right|_{\mid \Gamma_{1}}=h_{1}
\end{aligned}
$$

\section{Step 3:}

i) If the approximation $\left(u^{(2 k)}, \omega^{(2 k)}\right)$ is constructed, solve the two mixed wellposed boundary value problems:

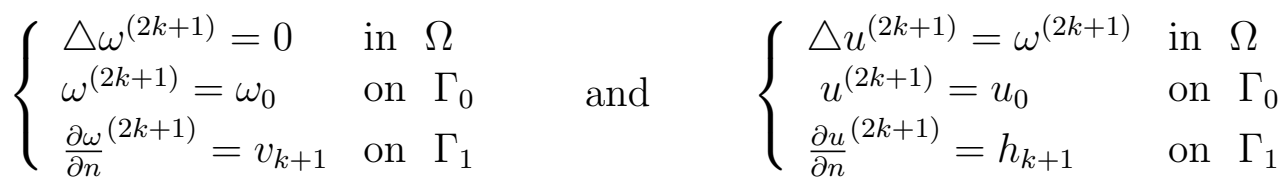

$$
\begin{aligned}
& \text { to obtain } \quad \omega_{\mid \Gamma_{1}}^{(2 k+1)}=\omega_{k+2} \quad \text { to obtain } \quad u_{\mid \Gamma_{1}}^{(2 k+1)}=u_{k+2}
\end{aligned}
$$

ii) If the approximation $\left(u^{(2 k+1)}, \omega^{(2 k+1)}\right)$ is constructed, solve alternatively the two mixed well-posed boundary value problems:

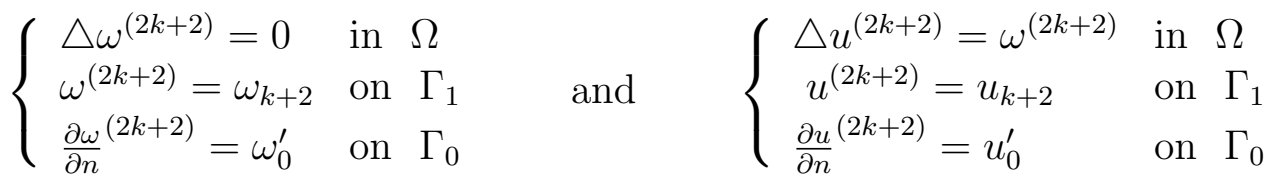

$$
\begin{aligned}
& \text { to obtain } \frac{\partial \omega^{(2 k+2)}}{\partial n}{ }_{\Gamma_{1}}=v_{k+2} \quad \text { to obtain } \quad \frac{\partial u}{\partial n}_{\mid \Gamma_{1}}^{(2 k+2)}=h_{k+2}
\end{aligned}
$$

Step 4: Repeat step 3 for $k \geq 0$ until a specified stopping criterion is satisfied.

This algorithm consists in constructing iteratively the traces which approximate the data on the part of the boundary $\Gamma_{1}$ of a function which verifies the biharmonic equation in the domain $\Omega$. 


\section{The boundary element method}

The boundary integral equations are classical kind of formulations for partial diferential equations [15], [17]. They have been used to solve a wide variety of real world problems.

In the presented iterative algorithm, we have to solve two types of mixed well-posed problems with Laplace and Poisson equation, where we will use the boundary elements method.

In order to give a brief description of boundary integral equations, we consider the following mixed boundary value problem:

Let $\Omega \subset \mathbb{R}^{2}$ of boundary $\Gamma=\Gamma_{0} \cup \Gamma_{1}$ such that $\Gamma_{0} \cap \Gamma_{1}=\emptyset$

$$
\begin{cases}\triangle \omega=0 & \text { in } \Omega \\ \omega=\omega_{0} & \text { on } \Gamma_{0} \\ q_{\omega}=\omega_{1} & \text { on } \Gamma_{1}\end{cases}
$$

where $q_{\omega}=\frac{\partial \omega}{\partial n}$ is the normal derivative of $\omega$.

The obtained integral equations for the function $\omega$ solution of mixt Laplace equation are:

$$
\begin{gathered}
\omega\left(x_{P}\right)+\int_{\Gamma}\left(\omega\left(x_{Q}\right) \frac{\partial G}{\partial n}\left(x_{P}, x_{Q}\right)-q_{\omega}\left(x_{Q}\right) G\left(x_{P}, x_{Q}\right)\right) \quad d \Gamma_{Q}=0 \quad x_{P} \in \Omega \\
\frac{1}{2} \omega\left(x_{P}\right)+\int_{\Gamma}\left(\omega\left(x_{Q}\right) \frac{\partial G}{\partial n}\left(x_{P}, x_{Q}\right)-q_{\omega}\left(x_{Q}\right) G\left(x_{P}, x_{Q}\right)\right) \quad d \Gamma_{Q}=0 \quad x_{P} \in \Gamma
\end{gathered}
$$

where $\omega$ is the solution of the mixed well-posed problem for Laplace equation and $G$ the fondamental solution of Laplace equation in $\Omega$ defined by: $G\left(x_{P}, x_{Q}\right)=\frac{1}{2 \pi} \ln \frac{1}{r\left(x_{P}, x_{Q}\right)} \quad$ avec $\quad r\left(x_{P}, x_{Q}\right)=\left\|x_{P}-x_{Q}\right\|_{2}$

We consider a subdivision of the domain boundary $\Omega$ in a finite number $N$ of boundary elements $\Gamma_{k}$, i.e. $\quad \Gamma=\cup_{k=1}^{N} \Gamma_{k}$.

We consider that the center of each element $\Gamma_{k}$ is designated as a collocation node $x_{k}$.

If we pose: $\frac{\partial G}{\partial n}\left(x_{P}, x_{Q}\right)=K^{d}\left(x_{P}, x_{Q}\right)$ and $G\left(x_{P}, x_{Q}\right)=K^{s}\left(x_{P}, x_{Q}\right)$

Then;

with the discretization described, we replace $x_{P}$ by $l$-th node $x_{l}$ where $l=$ $1, \cdots, N$, and the integral $\Gamma$ by a sum of integrals on $\Gamma_{k}$ for $k=1, \cdots, N$. On each element $\Gamma_{k}$ the functions $\omega$ and $q_{\omega}$ are constants i.e $\omega=\omega_{k}$ et 
$q_{\omega}=q_{\omega_{k}}$.

We obtain, $\quad \forall l, k=1, \cdots, N$

$$
\begin{aligned}
& \quad \frac{1}{2} \omega\left(x_{l}\right)+\sum_{k=1}^{N} \omega_{k} \underbrace{\int_{\Gamma_{k}} K^{d}\left(x_{l}, x_{Q}\right) d \Gamma_{Q}}_{H_{l k}}-\sum_{k=1}^{N} q_{\omega k} \underbrace{\int_{\Gamma_{k}} K^{s}\left(x_{l}, x_{Q}\right) d \Gamma_{Q}}_{G_{l k}}=0 \\
& \Leftrightarrow \quad \frac{1}{2} \omega\left(x_{l}\right)+\sum_{k=1}^{N} \omega_{k} H_{l k}-\sum_{k=1}^{N} q_{\omega_{k}} G_{l k}=0 \Leftrightarrow \underbrace{\frac{1}{2} \omega+H \omega}_{\widetilde{H} \omega}-G q_{\omega}=0 \\
& \widetilde{H} \text { and } G \text { are two square matrices of order } N . \\
& \omega=\left[\omega_{1}, \omega_{2}, \cdots, \omega_{N}\right]^{T} \in \mathbb{R}^{N} \text { the data of } \omega \text { on } \Gamma . \\
& q_{\omega}=\left[q_{\omega_{1}}, q_{\omega_{2}}, \cdots, q_{\omega_{N}}\right]^{T} \in \mathbb{R}^{N} \text { the data of } \frac{\partial \omega}{\partial n} \text { on } \Gamma .
\end{aligned}
$$

By rearranging the matrix system, such that all the unknowns on the right side and known ones the left side, we obtain the linear system:

$$
A x=\underbrace{\widetilde{G} b}_{f} \Leftrightarrow A x=f
$$

with;

$A$ and $\widetilde{G}$ are two square matrices of order $N$.

$x=\left[q_{\omega_{1}}, \cdots, q_{\omega_{m}}, \omega_{m+1}, \cdots, \omega_{N}\right]^{T} \in \mathbb{R}^{N}$ vector of unknown data.

$b=\left[\omega_{1}, \cdots, \omega_{m}, q_{\omega_{m+1}}, \cdots, q_{\omega_{N}}\right]^{T} \in \mathbb{R}^{N}$ vector of known data.

Solving this linear system allows us to find the unknown data on boundary.

Similarly, for the application of the BEM for the Poisson problem:

$$
\begin{cases}\triangle u=\omega & \text { in } \Omega \\ u=u_{0} & \text { on } \Gamma_{0} \\ q_{u}=u_{1} & \text { on } \Gamma_{1}\end{cases}
$$

where $q_{u}=\frac{\partial u}{\partial n}$ is the normale derivative of $u$. $\omega$ is the solution of the previous Laplace problem.

The only difference between the integral Laplace equation and Poisson one is that we must add the term $\omega$ of the second member, which gives:

- if $x_{P} \in \Omega$

$$
u\left(x_{P}\right)+\int_{\Gamma}\left\{u\left(x_{Q}\right) \frac{\partial G}{\partial n}\left(x_{P}, x_{Q}\right)-q_{u}\left(x_{Q}\right) G\left(x_{P}, x_{Q}\right)\right\} d \Gamma_{Q}+\int_{\Omega} \omega\left(x_{Q}\right) \cdot G\left(x_{p}, x_{Q}\right) d \Omega=0
$$


Vol. LV (2017) Iterative Method to Solve a Data Completion Problem... 137

- if $x_{P} \in \Gamma$

$$
\frac{1}{2} u\left(x_{P}\right)+\int_{\Gamma}\left\{u\left(x_{Q}\right) \frac{\partial G}{\partial n}\left(x_{P}, x_{Q}\right)-q_{u}\left(x_{Q}\right) G\left(x_{P}, x_{Q}\right)\right\} d \Gamma_{Q}+\int_{\Omega} \omega\left(x_{Q}\right) \cdot G\left(x_{p}, x_{Q}\right) d \Omega=0
$$

The term that we add is equal to:

$$
\int_{\Omega} \omega\left(x_{Q}\right) \cdot G\left(x_{p}, x_{Q}\right) d \Omega=\int_{\Gamma}\left\{\omega\left(x_{Q}\right) \frac{\partial F}{\partial n}\left(x_{p}, x_{Q}\right)-q_{\omega}\left(x_{Q}\right) F\left(x_{p}, x_{Q}\right)\right\} d \Gamma_{Q}
$$

where the function $F$ is required in this case of the fundamental solution of the biharmonic equation, i.e,

$$
F\left(x_{p}, x_{Q}\right)=-\frac{1}{8 \pi} r^{2}\left(x_{p}, x_{Q}\right)\left\{\ln r\left(x_{p}, x_{Q}\right)-1\right\}
$$

With the same notation and the same discretization of the boundary, we obtain: $\forall l, k=1, \cdots, N$

$$
\begin{aligned}
& \frac{1}{2} u\left(x_{l}\right)+\sum_{k=1}^{N} u_{k} \underbrace{\int_{\Gamma_{k}} K^{d}\left(x_{l}, x_{Q}\right) d \Gamma_{Q}}_{H_{l k}}-\sum_{k=1}^{N} q_{u k} \underbrace{\int_{\Gamma_{k}} K^{s}\left(x_{l}, x_{Q}\right) d \Gamma_{Q}}_{G_{l k}} \\
& +\sum_{k=1}^{N} \omega_{k} \underbrace{\int_{\Gamma_{k}} K^{b}\left(x_{l}, x_{Q}\right) d \Gamma_{Q}}_{D_{l k}}-\sum_{k=1}^{N} q_{\omega k} \underbrace{\int_{\Gamma_{k}} K^{a}\left(x_{l}, x_{Q}\right) d \Gamma_{Q}}_{C_{l k}}=0 \\
& \Leftrightarrow \quad \frac{1}{2} u\left(x_{l}\right)+\sum_{k=1}^{N} u_{k} H_{l k}-\sum_{k=1}^{N} q_{u k} G_{l k}+\sum_{k=1}^{N} \omega_{k} D_{l k}-\sum_{k=1}^{N} q_{\omega_{k}} C_{l k}=0 \\
& \Leftrightarrow \underbrace{\frac{1}{2} u+H u}_{\widetilde{H} u}-G q_{u}+D \omega-C q_{\omega}=0
\end{aligned}
$$

$\widetilde{H}, G, D$ et $C$ are square matrices of order $N$.

$$
\begin{aligned}
& u=\left[u_{1}, u_{2}, \cdots, u_{N}\right]^{T} \in \mathbb{R}^{N} \text { data of } u \text { on } \Gamma . \\
& q_{u}=\left[q_{u_{1}}, q_{u_{2}}, \cdots, q_{u_{N}}\right]^{T} \in \mathbb{R}^{N} \text { data of } \frac{\partial u}{\partial n} \text { on } \Gamma . \\
& \omega=\left[\omega_{1}, \omega_{2}, \cdots, \omega_{N}\right]^{T} \in \mathbb{R}^{N} \text { data of } \omega \text { on } \Gamma .
\end{aligned}
$$


$q_{\omega}=\left[q_{\omega_{1}}, q_{\omega_{2}}, \cdots, q_{\omega_{N}}\right]^{T} \in \mathbb{R}^{N}$ data of $\frac{\partial \omega}{\partial n}$ on $\Gamma$.

We rearrrange the matrix system, i.e. all the unknowns on the right side and knowns ones on the left side. We get the following linear system:

$$
A x^{\prime}=\underbrace{\widetilde{G} b^{\prime}-D \omega+C q_{\omega}}_{f^{\prime}} \Leftrightarrow A x^{\prime}=f^{\prime}
$$

with,

$A$ and $\widetilde{G}$ are two square matries of order $N$.

$$
\begin{aligned}
& x^{\prime}=\left[q_{u_{1}}, \cdots, q_{u_{m}}, u_{m+1}, \cdots, u_{N}\right]^{T} \in \mathbb{R}^{N} \text { vector of unknown data. } \\
& b^{\prime}=\left[u_{1}, \cdots, u_{m}, q_{u_{m+1}}, \cdots, q_{u_{N}}\right]^{T} \in \mathbb{R}^{N} \text { vector of known data. }
\end{aligned}
$$

\section{$5 \quad$ Numerical results}

The solution domain considered is an example with non-smooth boundary, which is a square $\Omega=(0,1) \times(0,1)$ with a boundary $\partial \Omega=\Gamma_{1} \cup \Gamma_{0}$ such that $\Gamma_{1} \cap \Gamma_{0}=\emptyset$.

where; $\Gamma_{1}$ is the underspecified boundary and $\Gamma_{0}$ is the overspecified boundary.

The analytical biharmonic function $u$ and the harmonic function $\omega$ which verified $\Delta u=\omega$ to be retrieved are given by:

$$
u(x, y)=\frac{x \sin (x) \cosh (y)-x \cos (x) \sinh (y)}{2}
$$

and

$$
w(x, y)=\cos (x) \cosh (y)+\sin (x) \sinh (y)
$$

The objective is to reconstruct the traces $u, \frac{\partial u}{\partial n}, \omega$ and $\frac{\partial \omega}{\partial n}$ of the functions $u$ and $\omega$. Hence, the convergence of the algorithm may be investigated by evaluating at every iteration the errors defined by:

$$
\begin{array}{cc}
\operatorname{Err}_{u}=\left\|u_{\text {num }}-u_{\text {ana }}\right\|_{2} & \operatorname{Err}_{Q u}=\left\|Q u_{\text {num }}-Q u_{\text {ana }}\right\|_{2} \\
\operatorname{Err}_{\omega}=\left\|\omega_{\text {num }}-\omega_{\text {ana }}\right\|_{2} & \operatorname{Err}_{Q \omega}=\| Q \omega \text { num }-Q \omega_{\text {ana }} \|_{2}
\end{array}
$$

In addition, we evaluated the following quantities:

$$
\mathrm{Con}_{u}=\left\|u_{\text {num }}^{(k+1)}-u_{\text {num }}^{(k)}\right\|_{2} \quad \operatorname{Con}_{Q u}=\left\|Q u_{\text {num }}^{(k+1)}-Q u_{\text {num }}^{(k)}\right\|_{2}
$$




$$
\operatorname{Con}_{\omega}=\left\|\omega_{\text {num }}^{(k+1)}-\omega_{\text {num }}^{(k)}\right\|_{2} \quad \operatorname{Con}_{Q \omega}=\left\|Q \omega_{\text {num }}^{(k+1)}-Q \omega_{\text {num }}^{(k)}\right\|_{2}
$$

In this study, we distinguish two different cases depending on the measure of the accessible part of the boundary.

First case: $\operatorname{mes}\left(\Gamma_{0}\right)=\operatorname{mes}\left(\Gamma_{1}\right)$

We consider that the underspecified boundary is given by $\Gamma_{1}=\{0\} \times(0,1) \cup$ $(0,1) \times\{1\}$ and the overspecified boundary $\Gamma_{0}=(0,1) \times\{0\} \cup\{1\} \times(0,1)$.

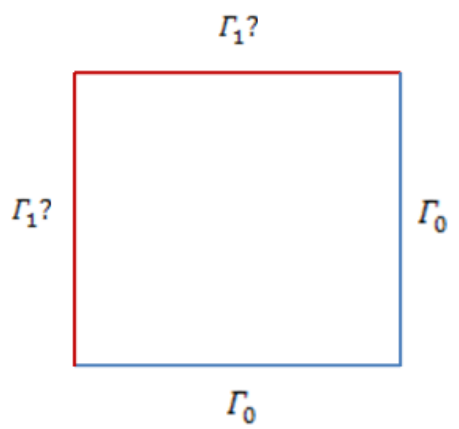

Figure 1: Representative schema of domain for case 1
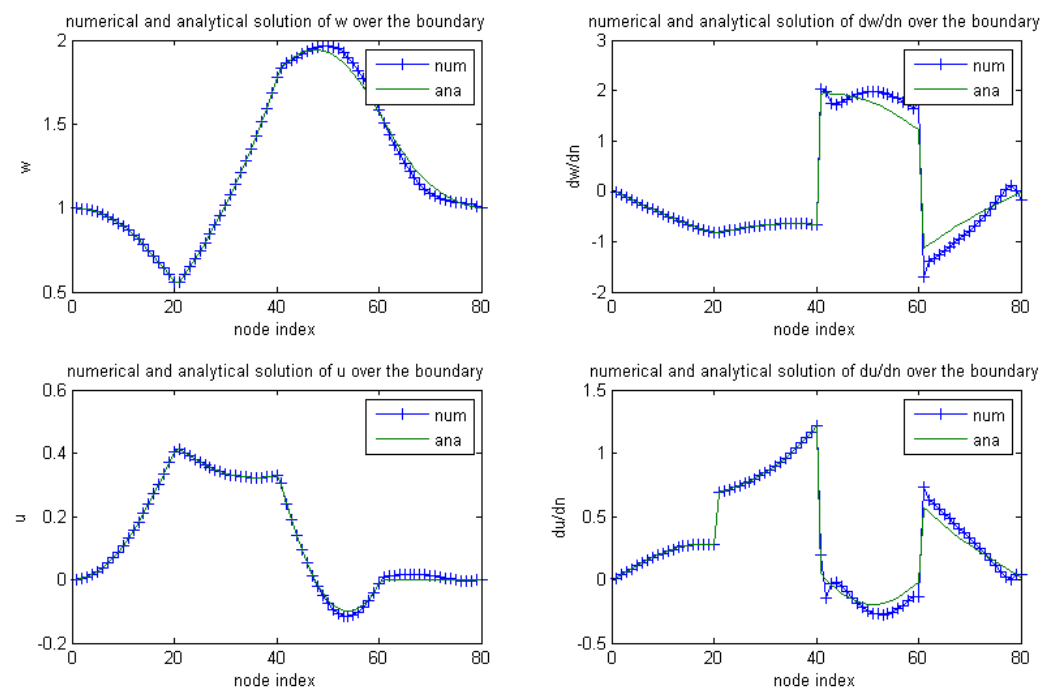

Figure 2: Comparison of the Dirichlet and Neumann boundary data for $u$ and $\omega$ with $N=80$ and $k=100$

The figure 2 presents results of the reconstructed data on the boundary compared with the exact solution with number of boundary elements $N=$ 
80, showing that they are accurate. The same results are obtained with $N=20,40$ showing that, more the number of elements increases, more the accuraty obtained is better.

Figure 3 (resp. figure 4) shows the evolution of the errors $\operatorname{Err}_{u}, \operatorname{Err}_{\omega}$, $\operatorname{Err}_{Q u}$ and $\operatorname{Err}_{Q \omega}$ (resp. Con ${ }_{u}, \operatorname{Con}_{w}, C \operatorname{Con}_{Q} u$ and $C_{0} n_{Q} w$ ) for each calculated data during the iterative process, showing that the error decreases in function of iterations and the error is smaller for Dirichlet conditions as that calculated for the Neumann conditions.

For the solution in the domain $\Omega$, we have obtained the result presented in figure 5 which shows that the approximate and the exact solutions are identical, allowing to conclude the performance of the iterative BEM algorithm that provides a good approximation for the biharmonic inverse problem.
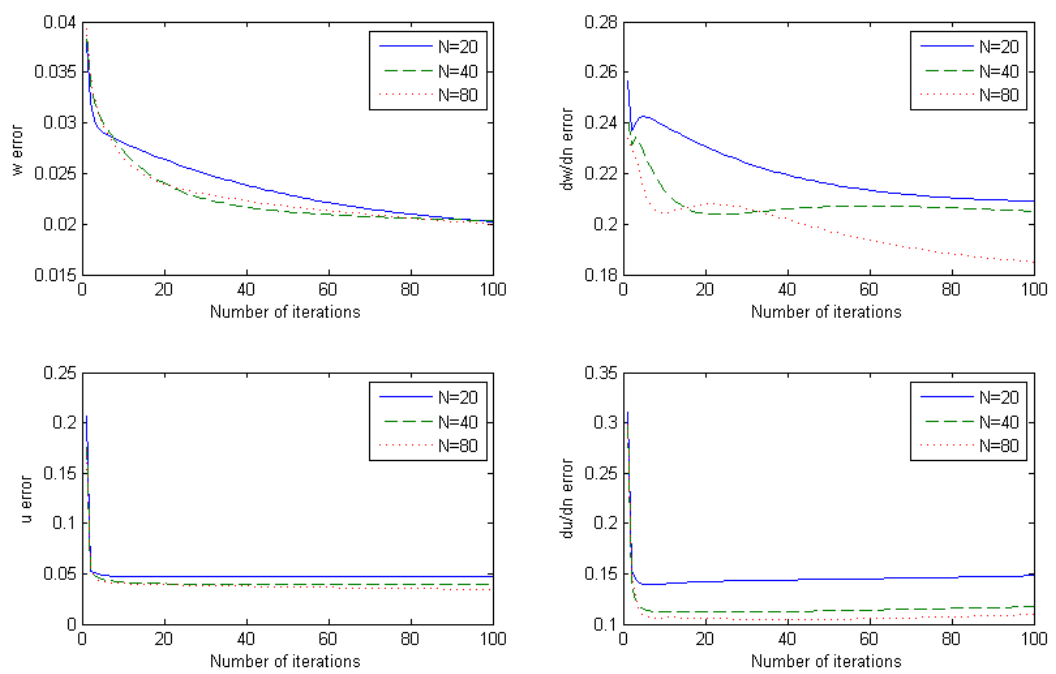

Figure 3: The errors $\operatorname{Err}_{u}, \operatorname{Err}_{\omega}, \operatorname{Err}_{Q u}$ and $\operatorname{Err}_{Q \omega}$ with $N=20,40,80$

Second case: $\operatorname{mes}\left(\Gamma_{0}\right)>\operatorname{mes}\left(\Gamma_{1}\right)$

We consider that the underspecified boundary is given by $\Gamma_{1}=\{0\} \times(0,1)$ and the overspecified boundary $\Gamma_{0}=(0,1) \times\{0\} \cup\{1\} \times(0,1) \cup(0,1) \times\{1\}$.

In this case, we note that the unknown boundary data obtained by the numerical algorithm are almost equal to the exact data. Comparing with the results obtained in the previous case, we can say that the results are more accurate when the unknown part is strictly smaller than the part that contains the known data.

To assess the accuracy of these results with respect to exact or analytical results, we calculate at each iteration $k$ the errors for each boundary data 
Vol. LV (2017) Iterative Method to Solve a Data Completion Problem... 141
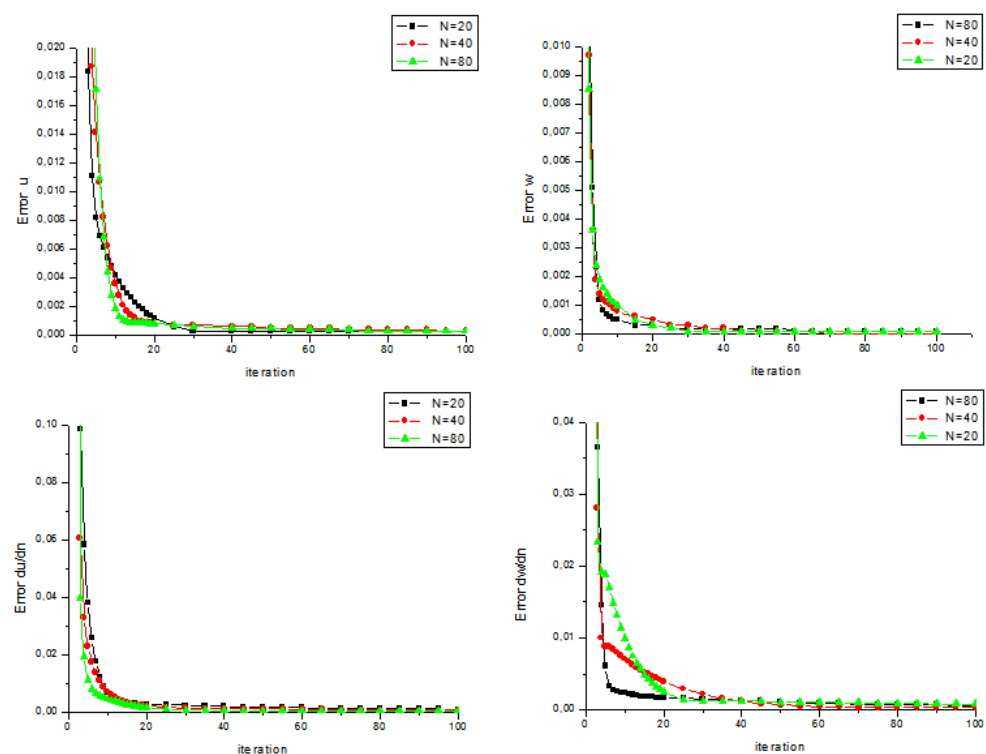

Figure 4: The Errors $\operatorname{Con}_{u}, \operatorname{Con}_{w}, \operatorname{Con}_{Q u}$ and $\operatorname{Con}_{Q w}$ with $N=20,40,80$
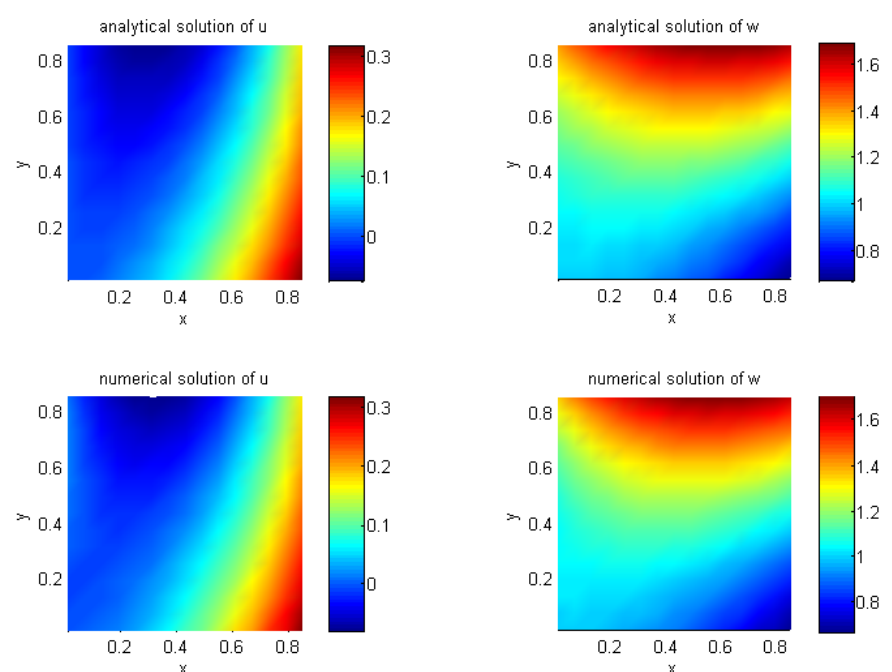

Figure 5: The exact solution and the approximate one with $N=40$ 


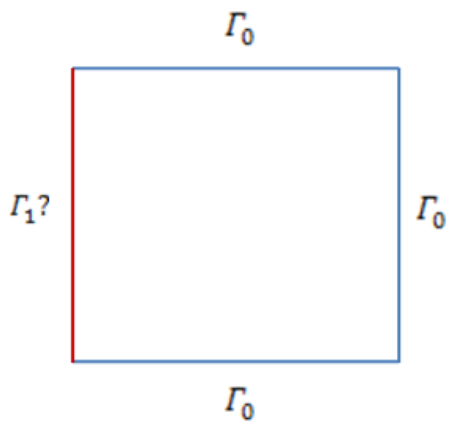

Figure 6: Representative schema of domain for case 2
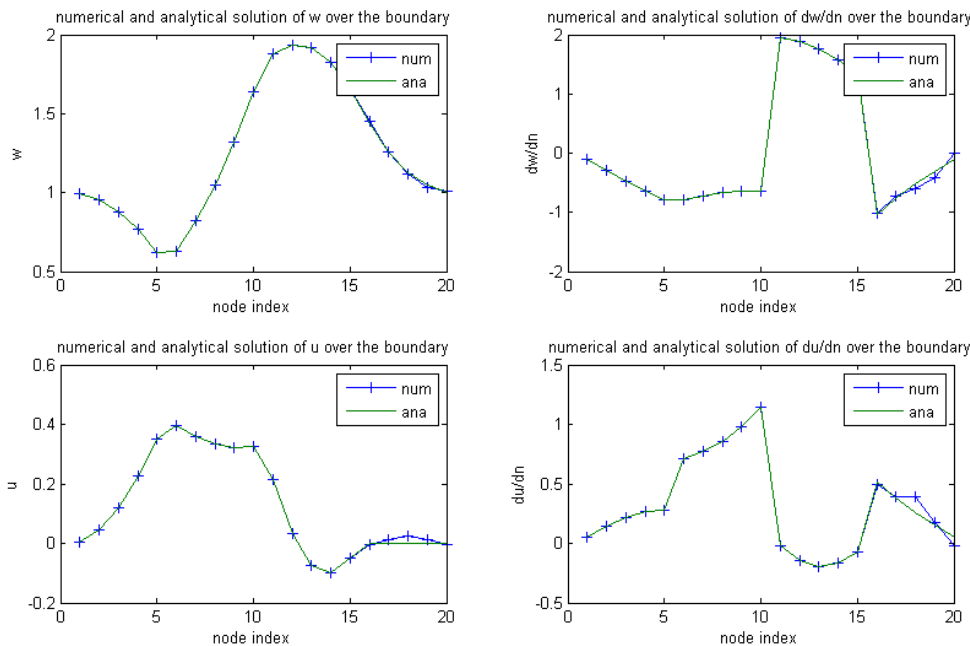

Figure 7: Comparison of the Dirichlet and Neumann boundary data for $u$ and $\omega$ with $N=20$ and $k=100$ 
Vol. LV (2017) Iterative Method to Solve a Data Completion Problem... 143
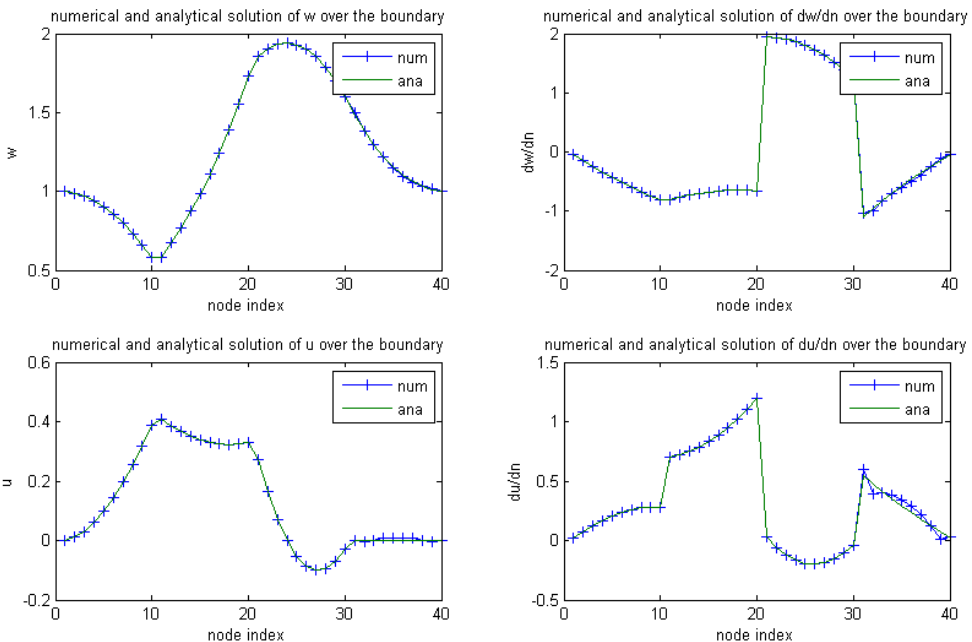

Figure 8: Comparison of the Dirichlet and Neumann boundary data for $u$ and $\omega$ with $N=40$ and $k=100$
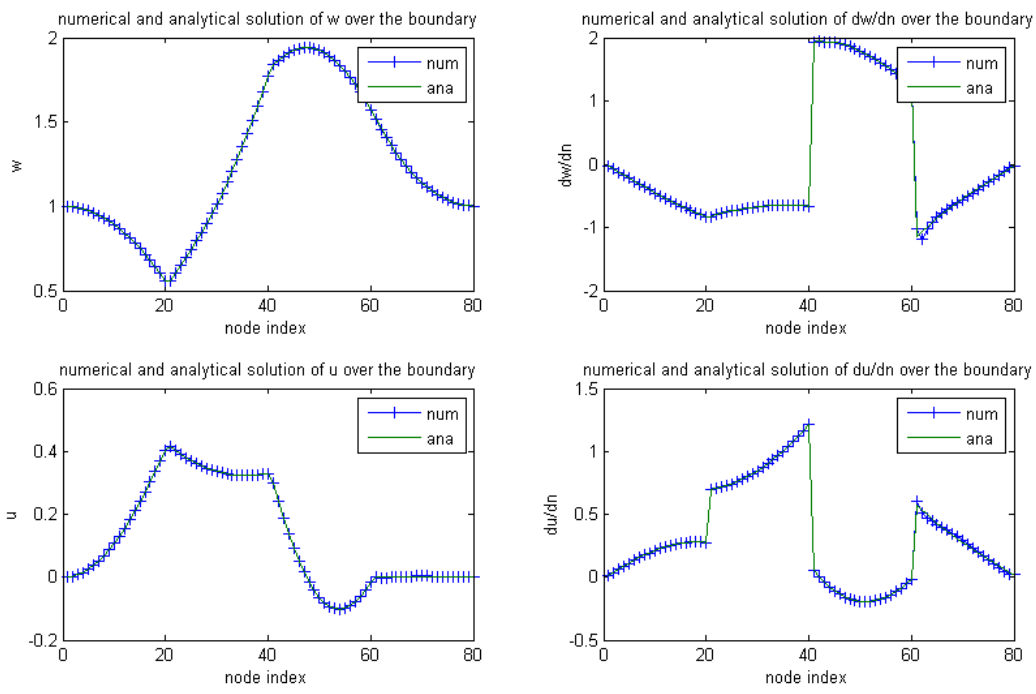

Figure 9: Comparison of the Dirichlet and Neumann boundary data for $u$ and $\omega$ with $N=80$ and $k=100$. 
$u, \frac{\partial u}{\partial n}, \omega$ and $\frac{\partial \omega}{\partial n}$ that we have already defined above by $\operatorname{Err}_{u}, \operatorname{Err}_{Q u}, \operatorname{Err}_{\omega}$ and $\operatorname{Err}_{Q \omega}$.

The obtained results of error for each boundary data for different values of $N$ are presented in figure 10 .
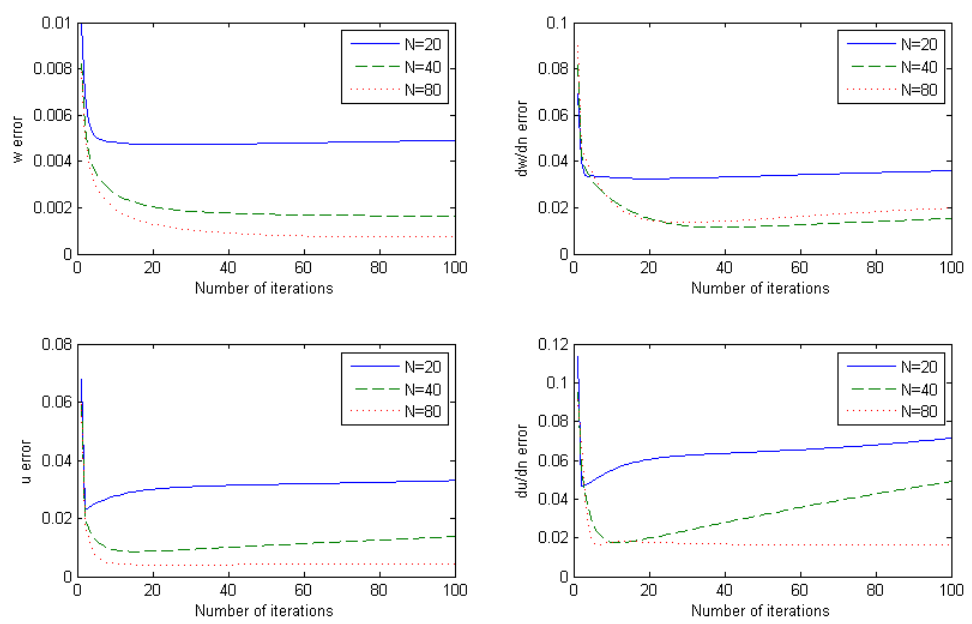

Figure 10: The errors $\operatorname{Err}_{u}, \operatorname{Err}_{\omega}, \operatorname{Err}_{Q u}$ and $\operatorname{Err}_{Q \omega}$ with different choices of $N=20,40,80$

During the iteration process, the calculated errors for the reconstructed data are much smaller when $N$ is greater. The value of the errors in this case is smaller than the value of the errors obtained in the first case.

According to the reconstructed data on the boundary, the solution inside the domain $\Omega$ with $N=40$ is given by figure 11 .

To study the stability of the obtained solutions, different perturbations were introduced in the prescribed data on the accessible part of the boundary to see their impact on the results. The results obtained are given in figure 12 .

\section{Conclusion}

In this work, a data completion problem for the biharmonic equation with four specific conditions on the inaccessible part of the boundary of rectangular domain is presented. In order to solve it, we proposed a factorization of the problem in two inverse problems for the Poisson and the Laplace equations 
Vol. LV (2017) Iterative Method to Solve a Data Completion Problem... 145
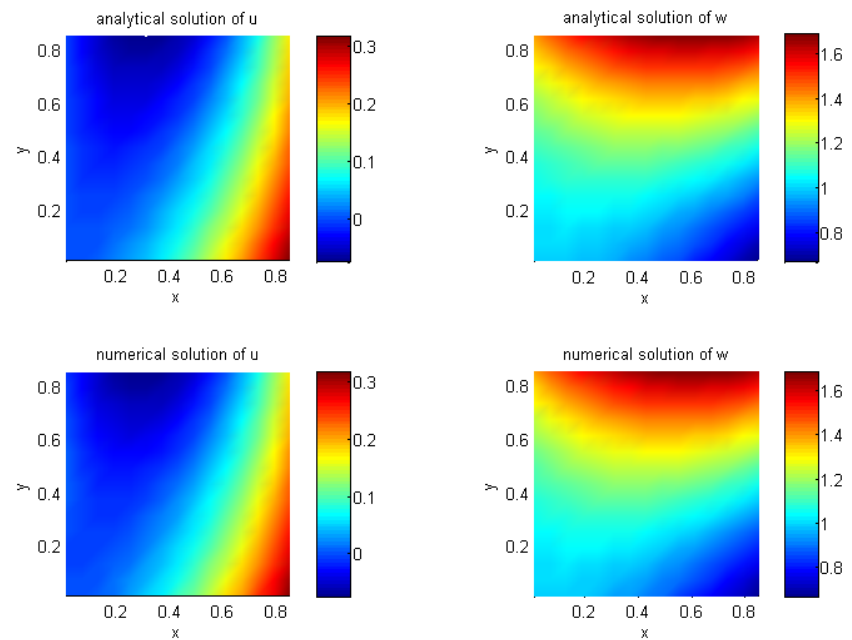

Figure 11: Comparison betwem the exact solution and the obtained one in the domain $\Omega$ with $N=40$.
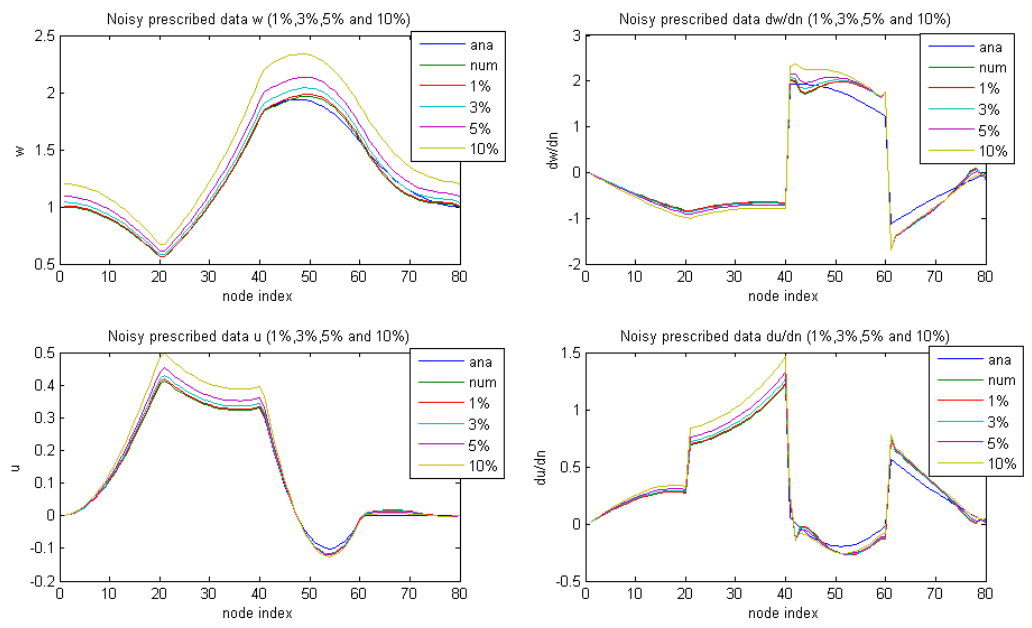

Figure 12: The obtained results with perturbations of conditions $1 \% \%, 3 \%$, $5 \%, 10 \%$ compared to analytical solution and non perturbed solution. 
which are solved by the iterative method developped by Kozlov, Mazya and Fomin using the BEM. It is shown that the iterative BEM algorithm produces a convergent and accurate numerical solution with respect to increasing the number of boundary elements and the number of iterations. Furthermore, the iterative alternating algorithm has a regularizing character and the stability of the numerical solution was shown by imposing various amounts of noise in the input data.

\section{References}

[1] B. Bialecki, A fourth order finite difference method for the Dirichlet biharmonic problem, Numerical Algorithms, 61(3), (2012), 351-375

[2] M. B. Artzi, J. P. Croisille, and D. Fishelov, A Fast Direct Solver for the Biharmonic Problem in a Rectangular Grid, SIAM J. Sci. Comput., 31(1), (2008), 303-333.

[3] M. M. Gupta and R. P. Manohar, Direct solution of the biharmonic equation using noncoupled approach, Journal of Computational Physics, 33(2), (1979), 236248.

[4] J. Hadamard, Lectures on the Cauchy Problem in Linear Partial Differential Equations, Yale University Press, New Haven, 1923

[5] R. V. Kohn and A. McKenney, Numerical implementation of a variational method for electrical impedance tomography, Inverse Problem, 6, (1990), 389-414

[6] R. Lattès and J. L. Lions, Méthode de Quasi-réversibilité et Applications, Dunod, Paris, 1967.

[7] L. Bourgeois, A mixed formulation of quasi-reversibility to solve the Cauchy problem for Laplace's equation, Inverse Problems, 21, (2005), 1087-1104.

[8] M. V. Klibanov and F. Santosa, A computational quasi-reversibility method for Cauchy problems for Laplace's equation, SIAM J.Appl. Math, 51, (1991), 1653-1675.

[9] F. Delvare, A. Cimetière, M. Jaoua, and F. Pons, Solution of the Cauchy problem using iterated Tikhonov regularization, Inverse Problems, 17, (2001), 553570 .

[10] V. A. Kozlov, V. G. Maz'ya, and A. V. Fomin, An iterative method for solving the Cauchy problem for elliptic equation, Comput. Maths. Math. Phys., 31(1), (1991), $45-52$.

[11] M. Jourhmane and A. Nachaoui, Convergence of an alternating method to solve the Cauchy problem for Poisson's equation, Applicable analysis, 81, (2002), 10651083.

[12] M. Jourhmane, D. Lesnic, and N. S. Mera, Relaxation procedures for an iterative algorithm for solving the Cauchy problem for the Laplace equation, Engineering Analysis with Boundary Elements, 18, (2004), 655-665. 
[13] C. Tajani and J. Abouchabaka, Missing boundary data reconstruction by an alternating iterative method, Int. Jour. of Adv. in Eng. and Tech., 2(1), (2012), $578-586$.

[14] C. Tajani, O. Abdoun, and J. Abouchabaka, Data Recovering Problem Using a New KMF Algorithm for Annular Domain., American Journal of Computational Mathematics, 2(2), (2012), 88-94.

[15] C.A. Brebia, J. C. F. Telles, and L. C. Wrobel, Boundary Element Techniques: Theory and Application in Engineering, Springer, Berlin, 1984.

[16] J. R. Cannon and M. M. Cecchi, The numerical solution of some biharmonic problems by mathematical programming techniques, SIAM J. Numer. Anal., 3, (1996), 451.

[17] D. Lesnic, L. Elliott, and D. B. Ingham, An alternating boundary element method for solving Cauchy problems for the biharmonic equation, Inverse Probl. Eng., 5, (1997), 145-168.

[18] D. Lesnic, L. Elliott, D. B. Ingham, and A. Zeb, A numerical method for an inverse biharmonic problem, Inverse Problems in Engineering, 7, (1999), 409-431.

[19] J. L. Lions and E. Magenes, Problèmes aux limites non homogènes et applications, Dunod, Paris, 1, 1968

Chakir Tajani

Department of Mathematics

Polydisciplinary faculty of Larache, Abdelmalek Essaadi University

Morocco

E-mail: chakir_tajani@hotmail.fr

Houda Kajtih

Faculty of Sciences and Techniques of Tangier, Abdelmalek Essaadi University

Morocco

E-mail: houda.kjt@gmail.com

\section{Ali Daanoun}

Faculty of Sciences and Techniques of Tangier, Abdelmalek Essaadi University

Morocco

E-mail: a_daanoun@yahoo.fr

Received: 7.11.2016

Accepted: 4.01.2017 